\title{
Preface to the Special Issue on Physical Mechanics
}

The 23rd International Congress of Theoretical and Applied Mechanics (ICTAM2012) will be held in Beijing, China, from August 19 to 24, 2012. The Congress (ICTAM), as the most authoritative and comprehensive conference in mechanics in the world and regarded as the "Olympic games" of mechanicians, is organized by the International Union of Theoretical and Applied Mechanics (IUTAM) every four years. It brings together scientists and students from all over the world to exchange state-of-the-art achievements in the field of mechanics to promote the research, application and education of mechanics. The ICTAM2012, hosted by the Chinese Society of Theoretical and Applied Mechanics (CSTAM), is expected to promote research and applications of mechanics in China as well as throughout the world. CSTAM has decided to launch a series of activities in 2012 named "Mechanics Year 2012", with the theme of "Mechanics · Achievement · Future".

To meet the magnificent ICTAM2012 and participate in the activities of the "Mechanics Year 2012", this special issue on "physical mechanics" is organized under the support of the Working Party of Physical Mechanics, CSTAM. This special issue gathers 30 papers which were all peer reviewed by at least two reviewers in accordance with the editorial policies of Science China: Physics, Mechanics and Astronomy.

In 1953, Dr. Hsue-Shen Tsien published a historical milestone paper entitled "Physical mechanics, a new field in engineering science" [1]. In this paper, Dr. Tsien indicated that the purpose of physical mechanics is to predict the engineering behavior of matter in bulk from the microscopic properties of its molecular and atomic constituents. The problems in physical mechanics can be classified into two categories: Problems in thermodynamic properties of matter at equilibrium, and problems in transport properties of matter not at equilibrium. Physical mechanics is a branch of engineering science mainly because its foremost purpose is in solving engineering problems.

Fifty nine years having passed since the publication of ref. [1], but the concept of physical mechanics of from-microscopic-to-macroscopic or bottom-up approach has shown its exuberant and incredible vitality. It has found applications in various scientific and engineering research fields, especially in micro- and nano-scale mechanics, surface and interface mechanics, soft matter mechanics, mechanical problems in micro- and nano-electromechanical systems (MEMS/NEMS) and high-temperature gas dynamics, although the term of "physical mechanics" may not be always actually adopted.

The 30 papers are presented in no particular grouping of topics in order to illustrate the interrelation between the diverse subjects. An effective method was proposed in ref. [2] to study the thermodynamic properties and constitutive relations of crystals at finite temperature based on the theory of lattice dynamics and interatomic potential of Embedded Atom Model (EAM). The diffusion, viscosity and thermal conductivity coefficients of gases between two parallel solid walls have been analytically obtained in ref. [3] based on the Green-Kubo relation under a hard-sphere model. Two special examples of using the physical mechanics approach to study the colloidal stability were presented in ref. [4]. Based on molecular force fields, a new finite element model was constructed in ref. [5] for multi-walled carbon nanotubes where the interlayer interactions and $\mathrm{C}-\mathrm{C}$ bonds were simulated by the elements of piecewise-linear spring and rectangular cross section beam, respectively. Shock tube study of kerosene ignition delay at high pressures was made experimentally in ref. [6]. Incorporating the contribution of solute atoms to the Helmholtz free energy of solid solution, a linear relation was derived between Young's modulus and the concentration of solute atoms in ref. [7]. The intrinsic origin of the exotic paradox of superplasticity, superelasticity and superrigidity demonstrated by solid sizing from monatomic chain to mesoscopic grain was investigated in ref. [8]. A stochastic-elasticity framework by which a type of materials with linearly graded elastic modulus can be designed to achieve "equal load sharing" and enhanced cooperative rebinding among interfacial molecular bonds was demonstrated in [9]. How the mechanical characteristics of human RBCs membrane are changed by $\mathrm{C}_{60}$ NPs infiltration was investigated based on atomic force microscopy combined with theoretical analysis in ref. [10]. A review of micro/nano technologies and theories for electroporation of biological cells was presented in ref. [11]. A micromechanical theoretical model of double-walled carbon nanotube pullout from a composite matrix was presented in ref. [12] with the interfacial residual stress and van 
der Waals force taken into account. The yielding behavior of copper nanowire in the presence of vacancies was studied by using molecular dynamics simulations in ref. [13]. Based on particle dynamic simulation and experimental observation, a detailed analysis on the mechanism of dust removal by an electric curtain was presented in ref. [14]. The key role played by the terminal film in a film-terminated fibrillar array was theoretically studied in ref. [15]. The unique determination of material elastoplastic properties by a sharp indentation was discussed in ref. [16]. The fabrication technique of micro/nano-scale speckle patterns with focused ion beam system for digital image correlation measurement via scanning electron microscopy was studied in ref. [17]. The effect of triple-phase contact line on contact angle hysteresis was studied both experimentally and theoretically in ref. [18]. The effects of agglomerates on the densification behavior and microstructural evolution during solid-state sintering of a cube of copper particles were studied with discrete element method in ref. [19]. The distribution of the local stress was studied in ref. [20] for the problem that a nonlocal bar is in uniform tension, where both the approximate analytical solution for the bi-exponential kernel and numerical solutions for the bi-exponential kernel and the Gaussian kernel were obtained for different values of the parameters. The interaction potential between micro/nano curved surface and a particle located inside the surface was investigated both theoretically and numerically in ref. [21,22]. The mechanical behavior of graphene under in-plane shear was studied using molecular dynamics simulations in ref. [23], which showed that the shear behavior of chiral graphene is dependent on the loading direction due to its structural asymmetry. A combination of high-resolution transmission electron microscopy, geometric phase analysis and numerical moiré method was used to investigate the deformation field of a micro-crack in single-crystal silicon in ref. [24]. The size-dependence for the cracks of drying particulate films fabricated by the controlled vertical drying deposition method was studied in ref. [25]. The uncertainty and the universality in the power-law singularity as a precursor of catastrophic rupture were studied in ref. [26]. Thermal vibration of single-layered graphene sheets was investigated in ref. [27] using plate model together with the law of energy equipartition and molecular dynamics (MD) method based on the condensed-phase optimized molecular potentials for COMPASS force field. The size effect on mechanical behavior of Au pillars was simulated by MD in ref. [28]. A theoretical ellipsoidal surface model was established for predicting the equilibrium shapes of the droplet in ref. [29]. An atomistic-continuum coupled model for thermo-mechanical behaviors of materials in nano-micro scales was presented in ref. [30]. Last but not least, $\mathrm{MD}$ and first-principle simulations were implemented in ref. [31] for the formation mechanism of $\mathrm{ZnO}$ diatomic chain and multi-shell structure.

In closing, I do hope that this special issue comprising 30 papers will serve as a fountainhead of new research ideas for physical mechanics, and concomitantly, new and exciting research progress will emerge in the near future. I would like to express my gratitude to all the authors, reviewers and editors for their contributions to this special issue.

Professor ZHAO YaPu Guest Editor

Institute of Mechanics, Chinese Academy of Sciences

April, 2012

1 Tsien H S. Physical mechanics, a new field in engineering science. J Am Rocket Soc, 1953, 23: 17-24

2 Tang Q H, Wang T C, Shang B S, et al. Thermodynamic properties and constitutive relations of crystals at finite temperature. Sci China-Phys Mech Astron, 2012, 55(6): 918-926

3 Fei F, Fan J, Jiang J Z. Solid wall effect on the transport coefficients of gases. Sci China-Phys Mech Astron, 2012, 55(6): 927-932

4 Sun Z W, Xu S H. Two examples of using physical mechanics approach to evaluate the colloidal stability. Sci China-Phys Mech Astron, 2012, 55(6): 933-939

5 Miao C Y, Li H J, Guo W L. Radial breathing modes of multi-walled carbon nanotubes by an atomic beam-spring model. Sci China-Phys Mech Astron, 2012, 55(6): 940-946

6 Liang J H, Wang S, Hu H H, et al. Shock tube study of kerosene ignition delay at high pressures. Sci China-Phys Mech Astron, 2012, 55(6): 947-954

7 Yang F Q. Diffusion-induced stress in inhomogeneous materials: concentration-dependent elastic modulus. Sci China-Phys Mech Astron, 2012, 55(6): 955-962

8 Ma Z S, Zhou Z F, Huang Y L, et al. Mesoscopic superplasticity, superelasticity, and superrigidity. Sci China-Phys Mech Astron, 2012, 55(6): 963-979

9 Zhang W L, Qian J, Yao H M, et al. Effects of functionally graded materials on dynamics of molecular bond clusters. Sci China-Phys Mech Astron, 2012, 55(6): 980-988

10 Zhang X Y, Zhang Y, Zheng Y, et al. Investigating effects of nano-particles infiltration on mechanical properties of cell membrane using atom force microscopy. Sci China-Phys Mech Astron, 2012, 55(6): 989-995

11 Lee Y K, Deng P G. Review of micro/nano technologies and theories for electroporation of biological cells. Sci China-Phys Mech Astron, 2012, 55(6): 996-1003

12 Zhou L J, Kang Y L, Guo J G. Theoretical model of double-walled carbon nanotube pullout from a composite matrix. Sci China-Phys Mech Astron, 2012, 55(6): 1004-1009 
13 Liu X M, Yang X B, Wei Y G. Yielding behavior of copper nanowire in the presence of vacancies. Sci China-Phys Mech Astron, 2012, 55(6): $1010-1017$

14 Sun Q X, Yang N N, Cai X B, et al. Mechanism of dust removal by a standing wave electric curtain. Sci China-Phys Mech Astron, 2012, 55(6): $1018-1025$

15 Yan S P, He L H, Wang H J. Adhesion hysteresis of a film-terminated fibrillar array. Sci China-Phys Mech Astron, 2012, 55(6): 1026-1031

16 Ma Z S, Zhou Y C, Long S G, et al. A new method to determine the elastoplastic properties of ductile materials by conical indentation. Sci China-Phys Mech Astron, 2012, 55(6): 1032-1036

17 Li Y J, Xie H M, Luo Q, et al. Fabrication technique of micro/nano-scale speckle patterns with focused ion beam. Sci China-Phys Mech Astron, 2012, 55(6): 1037-1044

18 Yu Y, Wu Q, Zhang K, et al. Effect of triple-phase contact line on contact angle hysteresis. Sci China-Phys Mech Astron, 2012, 55(6): 1045-1050

19 Wang C, Chen S H. The influence of agglomerates on the densification and microstructure evolution in sintering of a multi-particle system. Sci China-Phys Mech Astron, 2012, 55(6): 1051-1058

20 Zhu X W, Dai H H. Solution for a nonlocal elastic bar in tension. Sci China-Phys Mech Astron, 2012, 55(6): 1059-1065

21 Wu J Y, Yin Y J, Wang X G, et al. Interaction potential between micro/nano curved surface and a particle located inside the surface: (I) driving forces induced by curvatures. Sci China-Phys Mech Astron, 2012, 55(6): 1066-1076

22 Wu J Y, Yin Y J, Huang K, et al. Interaction potential between micro/nano curved surface and a particle located inside the surface: (II) numerical experiment and equipotential surfaces. Sci China-Phys Mech Astron, 2012, 55(6): 1077-1082

23 Yi L J, Chang T C. Loading direction dependent mechanical behavior of graphene under shear strain. Sci China-Phys Mech Astron, 2012, 55(6): 1083-1087

24 Zhao C W, Xing Y M. Quantitative analysis of nanoscale deformation fields of a crack-tip in single-crystal silicon. Sci China-Phys Mech Astron, 2012, 55(6): 1088-1092

25 Cao H, Lan D, Wang Y R. Crack and size-dependence of shear modulus in a drying particulate film. Sci China-Phys Mech Astron, 2012, 55(6): 1093-1097

26 Jin Y, Xia M F, Wang H Y. Uncertainty and universality in the power-law singularity as a precursor of catastrophic rupture. Sci China-Phys Mech Astron, 2012, 55(6): 1098-1102

27 Liu R M, Wang L F. Stochastically driven vibrations of single-layered graphene sheets. Sci China-Phys Mech Astron, 2012, 55(6): 1103-1110

28 Tang Q H. Effect of size on mechanical behavior of Au pillars by molecular dynamics study. Sci China-Phys Mech Astron, 2012, 55(6): 1111-1117

29 Wang X W, Yu Y. Analysis of the shape of heavy droplet on flat and spherical surface. Sci China-Phys Mech Astron, 2012, 55(6): 1118-1124

30 Xiang M Z, Cui J Z, Li B W, et al. Atom-continuum coupled model for thermo-mechanical behavior of materials in micro-nano scales. Sci China-Phys Mech Astron, 2012, 55(6): 1125-1137

31 Wang B B, Wang F C, Zhao Y P. Understanding formation mechanism of ZnO diatomic chain and multi-shell structure using physical mechanics: Molecular dynamics and first-principle simulations. Sci China-Phys Mech Astron, 2012, 55(6): 1138-1146 